Case Report

\title{
Endovascular stenting for treatment of superior vena cava syndrome
}

\author{
Subramanyam $K^{1}$, Janardhan Kamath ${ }^{2}$, Dilip Johny ${ }^{3} \&$ Paul T. Joyes ${ }^{4}$ \\ ${ }^{1}$ Professor \& HOD, ${ }^{3}$ Lecturer, Department of Cardiology, ${ }^{2}$ Associate Professor, Department of Nephrology, \\ ${ }^{4}$ Post Graduate, Department of M edicine, K. S. Hegde M edical College, \\ Nitte University, Mangalore, Karnataka, India. \\ Correspondence \\ Paul T. Joyes \\ Post Graduate, Department of M edicine, K. S. Hegde Medical College, Nitte University, M angalore 575018, Karnataka, India. \\ Mobile : +91 9880854869 E-mail : rush2paul.pj@gmail.com
}

\begin{abstract}
Superior vena cava syndrome (SVCS) is a well-known manifestation of benign and malignant tumors of the upper mediastinum. Superior vena cava (SVC) obstruction and thrombosis caused by indwelling venous catheters is a growing problem in patients on regular haemodialysis. We present a 62 -year-old woman with typical signs and symptoms of SVCS, secondary to thrombosis surrounding the indwelling central catheter who was treated with endovascular stenting by the percutaneous approach. We obtained both procedural success with complete restoration of blood flow and immediate relief of symptoms.
\end{abstract}

Keywords: SVCS, Endovascular stenting, thrombosis, central line

\section{Introduction}

Superior vena cava syndrome (SVCS) is a well-known manifestation of benign and malignant tumors of the upper mediastinum that causes obstruction of blood flow through the superior vena cava (SVC). Superior vena cava (SVC) obstruction and thrombosis caused by indwelling venous catheters is a growing problem in patients on regular haemodialysis and is associated with an appreciable morbidity and mortality. With the advances in interventional cardiology, percutaneous treatment by stenting has become a reasonable strategy in superior vena cava syndrome (SVCS), whether the underlying disease is malignant or benign. We present a 62-year-old woman with typical signs and symptoms of SVCS, who was treated with endovascular stenting by the percutaneous approach. We obtained both procedural success with complete restoration of blood flow Access this article online Quick Response Code

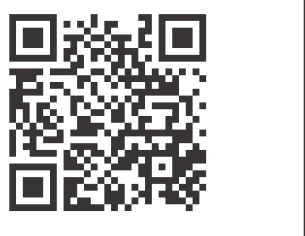
and immediate relief of symptoms.

\section{Case report}

A 62 year old lady with dialysis dependent renal failure was admitted in our hospital on13/2/2015 with history of progressive swelling of face, neck and upper chest since 1 month duration (Figure1). She was dialysed through a perm catheter on the right jugular which was inserted about 2 years back (Figure3). On evaluation she was diagnosed to have obstruction of SVC due to the thrombus surrounding the indwelling central line. A venogram done in our hospital revealed poor flow through the SVC. Echocardiogram done was also suggestive of decreased SVC flow. The perm catheter had very poor flow and hence a Right femoral trialyser was inserted for dialysis. Patient was posted for superior vena caval stenting on 19/2/2015.

Superior vena cavography was performed via a right retrograde venous approach from the common femoral vein to evaluate the degree of SVC obstruction and it showed significant narrowing of the lumen with almost complete restriction of blood flow to the right atrium (Figure5). $0.035^{\prime \prime} \times 260 \mathrm{cms}$ Cordis Emerald guidewire was placed through the Perm catheter across SVC, RA and placed in IVC. Perm catheter was removed. Predilatation done with Admiral Xtreme $5 \times 80 \mathrm{~mm}$ Percutaneous transluminal angioplasty (PTA) balloon at 8 atmospheres. $16 \times 60 \mathrm{~mm}$ Self expanding (Boston Scientific) Wall stent was deployed. Post dilated with $8 \times 37 \mathrm{~mm}$ EV3 
balloon at 8 atmospheres. Immediate end result was good (Figure6). On the next day patient was completely relieved of her symptoms and her swelling of face and neck had completely resolved (Figure2). A screening echo was done which showed adequate flow through the SVC. Patient was started on anticoagulation with the target to keep the INR between $2-3$.
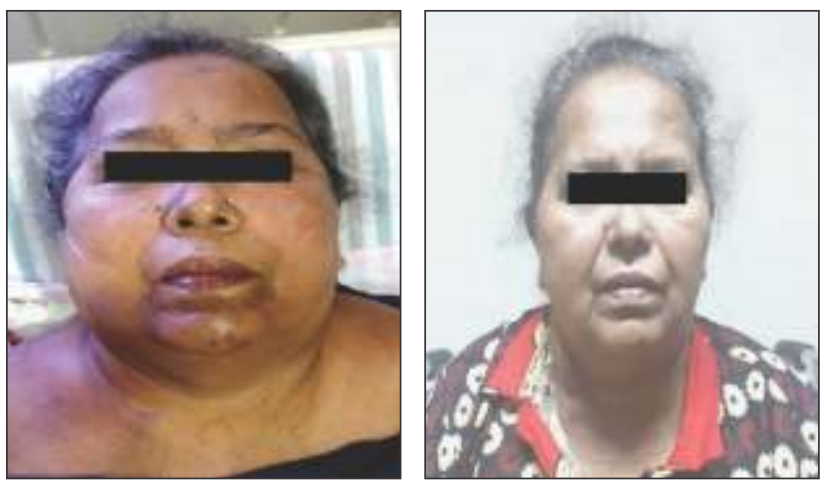

Figure 1: Patient before Figure 2: Patient after 12hrs of endovascular stenting of endovascularstenting Superior venacava

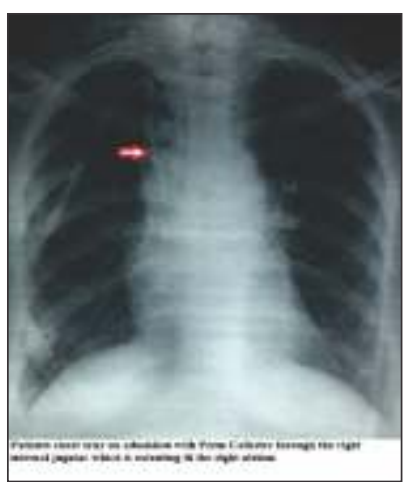

Figure 3 : The red arrow shows perm catheter in situ

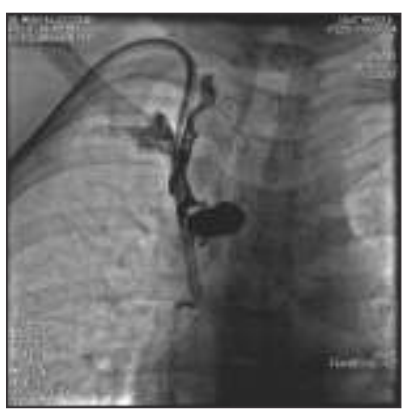

Figure 5 : Shows complete occlusion of superior venacava

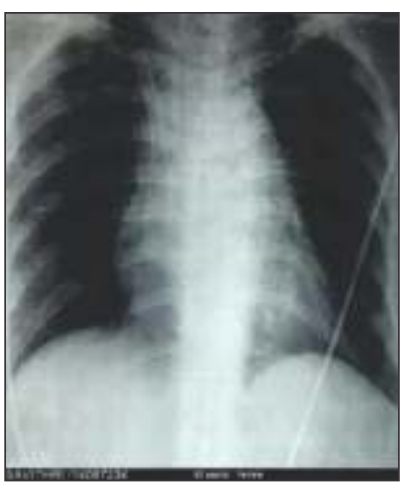

Figure 4 : Endovascular stent in Superior venacava extending into the Right atrium

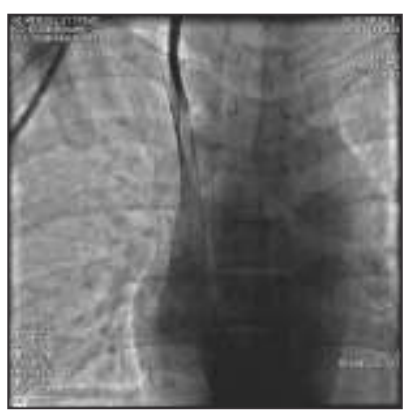

Figure 6 : Shows S/P endovascular stenting of superior venacava with patent stent extending to Right atrium

\section{Discussion}

Superior vena cava syndrome generally occurs as a result of either compression by an adjacent tumour in $85 \%$ of cases or compression by mediastinal lymph nodes. The clinical presentation of SVCS depends primarily on the acuteness of SVC obstruction. [1] The most common symptoms of presentation include facial and neck swelling, bilateral upper extremity swelling, dyspnea, headache, and cough. Superior vena cava syndrome is often clinically diagnosed, with the patient presenting with signs and symptoms related to venous congestion. [2]

Superior vena cava (SVC) obstruction and thrombosis caused by indwelling venous catheters is a growing problem, and is associated with an appreciable morbidity and mortality. [3]

Factors like under-provision of vascular access surgery, late referral and co-morbidities, large number of patients are subjected to use tunnelled venous catheters in the medium to long term haemodialysis. [4] One consequence of this will be the increasing incidence of central venous stenosis, thrombosis and exhaustion.

In this patient SVC obstruction was caused by thrombosis of SVC produced by venous catheter inserted for haemodialysis.

The pathophysiology is thought to be secondary to early intimal injury associated with focal endothelial denudation occurring with short-term central venous catheters and related to the position of the tip of the catheter, the site of insertion, the material, and predisposition to thrombosis.[5]With long-term catheter use, there is vein wall thickening, increased smooth muscle cells and focal catheter attachments to the vein wall with thrombus and collagen.[6] Management needs to be individualized. In the first few days of SVC thrombosis, removal of catheter, chemical or mechanical thrombolysis of the clot and/or venoplasty and stenting has been reported to resolve the symptoms. [7]

The use of angioplasty and stenting in the treatment of SVCS has developed over the past 15 years. With high 
success rates of stenting and nearly complete and immediate relief of symptoms, endovascular treatment has become a safe, consistent, and cost effective treatment for patients with SVCS. [8]

The most common complication of this therapy is stent thrombosis, stent migration perforation and rupture of veins which can be successfully treated with thrombolysis or stent replacement and anti-coagulation with warfarin. The chance of stent migration and rupture of veins is very rare. [9]

\section{References}

1. Rice TW, Rodriguez RM, Light RW. The superior vena cava syndrome: clinical characteristics and evolving etiology. M edicine 2006;85:37-42.

2. Lanciego C, Chacón JL, Julián A, Andrade J, López L, Martinez B, et al. Stenting as first option for endovascular treatment of malignant superior vena cava syndrome. AJ RAmJ Roentgenol 2001;177:585- 93.

3. Gopaluni $S$, Warwicker $P$.Superior vena cava obstruction presenting with epistaxis,haemoptysis and gastro-intestinal haemorrhage in two men receiving haemodialysis with central venous catheters: two case reports. Journal of M edical Case Reports 2009, 3:6180

4. Forauer AR, Theoharis $\mathrm{C}$ : Histologic changes in the human vein wall adjacent to indwelling central venous catheters. J Vasc Interv Radiol 2003, 14:1163.

5. Campisi C, Biffi R, Pittiruti M: Catheter-related central venous thrombosis: The development of a nationwide consensus paper in Italy. JAVA 2007, 12(1):39-45.

6. Rose SC, Kinney TB, Bundens WP, Valji K, Roberts AC: Importance of

\section{Conclusion}

Indwelling Central venous catheter is known to cause SVCS secondary to stent thrombosis.

Endovascular stenting for superior vena cava syndrome has shown rapid relief of symptoms after the procedure. This procedure is relatively safe and complication like stent migration and rupture of veins are very rare. Patients should be started on anti-coagulation with warfarin to prevent stent thrombosis.

Doppler analysis of transmitted atrial waveforms prior to placement of central venous access catheter. J Vasc Interv Radiol 1998, 9:927.

7. Carcao MD, Connolly BL, Chait P, Stain AM, Acebes M, Massicotte P, Blanchette VS: Central venous catheter-related thrombosis presenting as superior vena cava syndrome in a haemophilic patient with inhibitors. Haemophilia 2003, 9(5):578-583.

8. Rizvi AZ, Kalra M, Bjarnason H, Bower TC, Schleck C, Gloviczki P. Benign superior vena cava syndrome: stenting is now the first line of treatment. J Vasc Surg 2008;47:372-80.

9. Smayra T, Otal P, Chabbert V, Chemla P, Romero M, J offre F, et al. Longterm results of endovascular stent placement in the superior caval venous system. Cardiovasc Intervent Radiol 2001;24:388-94.

10. Entwisle KG, Watkinson AF, Reidy J. Case report: migration and shortening of a self-expanding metallic stent complicating the treatment of malignant superior vena cava stenosis. Clin Radiol 1996;51:593-5. 